\title{
Study on Application of Genre Analysis Tasks in Academic Writing for Chinese EFL Graduate Learners*
}

\author{
Hua $\mathrm{He}$ \\ School of Foreign Languages \\ Northwest University \\ Xi'an, China 710127
}

\begin{abstract}
One of the usual practices for teaching L2 learners' English academic writing is to raise learners' rhetorical consciousness. The author designs and carries out genre analysis tasks concerning Introduction, Body, Conclusion and Abstract of research articles in English Academic Writing course for Chinese EFL graduate learners. It's found that these tasks are helpful in raising learners' rhetorical consciousness and most learners are capable and cooperative in dealing with these tasks, while factors like subject difference, individual motivation and language proficiency affect task performance, upon which pedagogical implications are drawn to help promote academic English teaching for Chinese EFL graduate learners.
\end{abstract}

Keywords-genre analysis task; academic writing; graduate learners; Chinese EFL learners

\section{INTRODUCTION}

Genre-based approaches are not only widely used in understanding research articles, but also provide major pedagogical bases for "rhetorical consciousness raising" [1] p13. One of the usual practices for teaching L2 graduate learners' English academic writing is to raise learners' rhetorical consciousness [2] ${ }^{\mathrm{p} 85}$. Thus, it's of great significance for teachers to design and carry out proper genre analysis tasks for learners to achieve rhetorical consciousness and eventually apply those text and language features into their own construction of texts. These tasks, like what Swales defines, are relatable to genre acquisition within a discourse community, focusing learners on both the rhetorical organization and language features of the texts [3] $p$ 76, 82. Out of the three main approaches to genre, "English for Specific Purposes" (ESP) approach to teaching academic writing is arguably the most influential one in the context of teaching discipline-specific writing to L2 students in academic settings" [2] ${ }^{\mathrm{p} 85}$, upon which the present study is based.

In previous studies of teaching academic writing for graduate learners, there're not much researches focusing on ESP genre-based instruction. In addition, some researches (eg. Cheng) [4] are mainly based on case studies with very

*Fund: Graduate School of Northwest University, China: Course Construction of Academic Paper Writing (YKC17011). limited students from English-speaking countries. There are even less studies on graduate students from various disciplines outside the English speaking world in which English is their foreign language [2] ${ }^{\mathrm{p} 85}$. In this article, the author tentatively designs and carries out genre-analysis tasks in teaching academic writing course for Chinese EFL graduate learners (English as a Foreign Language). Furthermore, it's of growing interest in ESP and L2 writing to know how students respond and learn to produce academic genres [5] ${ }^{\mathrm{p} 52}$. So the author intends to know the learners' responses to genre analysis tasks by addressing the following questions: 1) Are genre analysis tasks helpful in raising Chinese EFL graduate students' rhetorical consciousness? 2) Are the students capable of dealing with these tasks? 3) What are the students' opinions towards these tasks adopted in Academic Writing course?

\section{THE STUDY}

\section{A. The Course}

The course is "Academic Writing", an optional course for first year master's students from a comprehensive university in China where the author teaches. The course aims at improving the students' academic writing ability and getting prepared for academic publication, especially for publishing international journal and conference papers. The teacher adopts an ESP genre-based approach, focusing on analyzing discipline-specific features of text construction and language expression. The course runs over a period of 18 weeks. Within the 1st two weeks, the students are asked to come up with a title of their future research article by negotiating with their tutors and fellow students, under which they're to write a 2000-3000 word article and hand in at the end of the semester. On the 3rd week, the students are asked to select one piece of journal or conference article within their own discipline for genre analysis by meeting 3 requirements: it's written by native English writer, selected from one of the top international journals or conferences and published within the latest three years. These requirements are to ensure a higher reliability of articles for students' analysis and adoption of both structure and language features in the construction of their own papers. From the 4th week on, the 
students start 4 series of genre analysis tasks concerning 4 main sections of research articles: Introduction, Body, Conclusion and Abstract, with each section covering a period of two or three weeks. The rest of the scheduled weeks are for the affiliated parts of research articles and language practice. At the end of each series of genre-analysis tasks, the students are asked to write a corresponding section of research paper under their chosen titles. At the end of the semester, the students need to compose a complete article out of these 4 parts written beforehand. The evaluation of the course consists of 2 parts, of which $60 \%$ is from course achievement test given by the course teacher and $40 \%$ is from English proficiency test at the university level for all first year master's students. Course achievement test accounting for $60 \%$ is composed of 3 parts: $20 \%$ is from class attendance and performance, another $20 \%$ from final test with questions and translation (questions are to test learners' theoretical and practical knowledge about academic paper writing and translation is to test learners' mastery of using academic English language), and the last 20\% from final research paper mentioned above.

\section{B. Genre Analysis Tasks}

Inspired by the "examine and discover" strategy [4] p290 and following principles for task design of genre-based pedagogy [6] ${ }^{\text {2238 }}$, genre-analysis tasks are designed in the present study based on a need analysis of the graduate students through the author's interview with students and their tutors from different schools and departments of the university where the author teaches. For each section of Introduction, Body, Conclusion and Abstract, a similar series of tasks are carried out in 5 stages, as shown in "Table I".

TABLE I. FIVE STAGES FOR EACH SERIES OF TASKS

\begin{tabular}{|l|l|l|l|}
\hline Stage & \multicolumn{1}{|c|}{ Task } & \multicolumn{1}{|c|}{$\begin{array}{c}\text { Role- } \\
\text { oriented }\end{array}$} & $\begin{array}{c}\text { In- or } \\
\text { Out- of } \\
\text { Class }\end{array}$ \\
\hline 1 & sample reading + discussion & students & in \\
\hline 2 & $\begin{array}{l}\text { move analysis }: \text { theory }+ \\
\text { practice }\end{array}$ & teacher & in \\
\hline 3 & coding + marking & students & out \\
\hline 4 & presentation & students & in \\
\hline 5 & revision + writing & students & out \\
\hline
\end{tabular}

In the 1st stage, the teacher gives sample reading to the students as an initiator of the discussion followed, the goal of which is to activate the students' previous knowledge of genres so as to be more motivated during the second stage when the teacher illustrates the method of move analysis. In stage 3 , the students are asked to code their articles chosen beforehand with moves and steps and mark language signals they've discovered corresponding to each move or step. In stage 4 , the students present in the class what they've coded and marked, followed by teacher and fellow students' comment and questions. In stage 5, the students revise their previous work and construct their own texts. Discussion in the 1st stage and presentation in the 4th are carried out in groups, and the coding work in the 3rd stage and writing in the 5 th are performed individually.

The advantages of these series of tasks are as follows. First, the alternate patterns of task forms like in- and out-of class as well as group and individual alternations help reinforce the students' concept of discipline-specific genres and raise rhetorical consciousness. This variety of alternations will not bore the students in addressing one problem during 2 or 3 weeks' time. Instead, it repeatedly exposes them to various task forms but focusing on one piece of genre-specific knowledge. This focused attention will undoubtedly help raise the students' rhetorical consciousness. Second, an integration of reading and writing is helpful for the students' internalization as well as adoption of rhetorical and linguistic features. The students' need for text construction enhances their initiative in reading, which will intrigue more cognitive reactions in examining and discovering discipline-specific features. These active cognitive reactions will help the students to better internalize rhetorical knowledge and a better internalization contributes to better adoption of rhetorical features in their own text construction.

\section{Participants}

99 Chinese EFL graduate students took this optional English course in the spring semester of 2018 from March to June. 96 of them joined the present study. Among these 96 participants, 19 of them are from art with 16 majoring in Economics and Management, 1 in Chinese Literature, 1 in Philosophy and 1 in Journalism, 24 are from science with 21 majoring in Mathematics and 3 in Physics and 56 are from engineering with 43 majoring in Information Science and Technology, 12 in Environmental Engineering and 1 in Chemical Engineering. Since this course is optional, this disciplinary distribution reveals the variation of students' needs for English academic paper writing, with engineering students at the highest level of need and art students at the lowest. All the students attend this course 2 hours a week for 18 weeks. Among them, only $18.8 \%$ of them have had academic writing course from field experts before taking this one in graduate study, which means this course is new to most participants. As to requirements for English paper publication, $46.9 \%$ of the students say it's required by their schools or supervisors, $33.3 \%$ say it's better than not to publish and only $19.8 \%$ say it's not required, showing that most students have a comparatively strong motivation to learn.

\section{Data-collection and Analysis}

After application of genre analysis tasks for each section of Introduction, Body, Conclusion and Abstract, the participants answer a questionnaire online (See Appendix 1) with 25 choice questions during class time. Based on Wen's data classification [7] ${ }^{\mathrm{p} 116}$, the author collects 4 categories of information as follows with one example following each list.

\section{1) Participants' Background Information (Questions 1-8)}

E.g. Does your school require you to publish one or more English academic papers before graduation?

2) Participants' acquired knowledge or skills after taking genre analysis tasks(Questions 9-14) 
E.g. Do you have a better mastery of the rhetorical pattern of the Introduction part after taking genre-analysis tasks?

3) Participants' capability in dealing with these tasks (Questions 15-21)

E.g. How difficult is it for you to analyze the moves of the Abstract part?

4) Participants' opinion towards these genre analysis tasks (Questions 22-25)

E.g. Do you think move-analysis is helpful for you to know the structure of research articles?

96 participants submitted the questionnaire. In all, the initial data of the present study comes mainly from the questionnaire of 96 participants, the author's observation log based on class observation and interviews with some students as supplementary. A quantitative method of analysis is adopted.

\section{FINDINGS AND DISCUSSION}

This section follows the sequence of 3 research questions raised in the Introduction part. Under each question, an overview of all students' responses is mentioned and then followed by the illustration of subject difference among 3 areas of art, science and engineering.

\section{A. The Influences of Genre-analysis Tasks in Raising Chinese EFL Graduate Students' Rhetorical Consciousness (Questions 9-14)}

First, when asked if they have a clear picture of rhetorical pattern for each part of research articles after taking related genre-analysis tasks (Q 9-12), the students choose 1 from 5 scales of "clearness" (very clear, clear, medium level, ambiguous, very ambiguous). For the "very clear" level, $43 \%$ of the participants feel so for Introduction, $29 \%$ for Body, $40 \%$ for Conclusion and $50 \%$ for Abstract. For the "clear" level, $56 \%$ is for Introduction, $60 \%$ for Body, $54 \%$ for Conclusion and $48 \%$ for Abstract. For the "medium level", only $1 \%$ is for Introduction, $10 \%$ for Body, $6 \%$ for Conclusion and $2 \%$ for Abstract. For "ambiguous" and "very ambiguous" levels, there are 0 responses. It is seen that most students are clear about rhetorical structure of research articles and a very low percentage of them remain unclear, so these genre-analysis tasks are helpful in raising their rhetorical consciousness. Besides, at the "very clear" level, the percentages of Abstract and Introduction parts are comparatively higher while those of the Body part is lower. The possible reason is, the moves and steps of Introduction and Abstract are easier to acquire because they are comparatively neat and similar across disciplines, while Body part is more complex and varied. For example, science and engineering focus more on the experiment, including pre-, in- and post-experimental related process, but art focuses on argumentation like background overview, contentious point, author's position, etc. Even within one discipline, variation and complication of particular research design might cause more or less differences. So lack of a universal pattern brings ambiguity.

Second, when asked if move analysis through sample reading helps the students in constructing their own texts $(\mathrm{Q}$ $13), 47 \%$ of them respond with "very great", $45 \%$ with "great", $8 \%$ with "medium level" and none with "no" or "not at all". And when asked if analysis of language signals corresponding to each move or step helps them with language expression in their own text construction (Q 14), $31 \%$ respond with "very great", $59 \%$ with "great", $9 \%$ with "medium level" and none with "no" or "not at all." It's shown that most students can apply what they have noticed into their own construction, which means these genreanalysis tasks are helpful in constructing their own texts. It's also noticed that only $31 \%$ of the students think it is of "very great help" for them to use the language expressions they've noticed into their own writings. From interview and class observation, the author finds out it results from syntactic difficulty. Some students say that it's easier to adopt lexical expression into their own writings, but the complexity of long and technical sentences sometimes prevent them from fully understanding and adoption. So teachers are suggested to give more attention on syntactic instruction in sample analysis.

As to subject difference, firstly, concerning if they have a clear picture of rhetorical patterns (Q 9-12), it's observed that the percentage of art students remain the highest on the level of "clear", while science and engineering students have similar results. On "medium" level, art students dwell on Body part while science students on Body, Conclusion and Abstract with Conclusion having the highest portion, and engineering students on all the 4 sections with Body part having the highest portion. We can see that most art students have a clear rhetorical picture but many haven't reached the level of "very clear". Engineering students go to the two extremes of "very clear" and "medium", especially for Body part. Science students have the same situation except that the percentage of "very clear" is not as high as that of engineering students. Reasons might lie in that, in the university where the author teaches, art students aren't required to publish English research articles during their stay in school, so their motivation of learning isn't very strong, but after one semester's learning, they've obtained a general picture, which explains why their percentage of "clear" is high but low on "very clear". For science and engineering students, some have publication requirements while others not, depending on disciplinary or supervisor requirements. That's why some are "very clear" while others remain at the "medium" level. So publication requirements and individual motivation play an important role. In addition, for students with comparatively weaker motivation like art students and those science and engineering students who're not required to publish, the difference lies probably in language proficiency. It's observed in class that art students have a better language performance than science and engineering students. So language barriers plus "no publication requirements" let some of the engineering students stay at the "medium" level. Secondly, when asked if move analysis helps them in their own construction (Q 13), 52\% of the 
engineering students choose "very helpful" and 52\% science students choose "helpful". When asked if analysis of language signals helps them with their own construction (Q 14 ), $68 \%$ of the art students, $65 \%$ science students and $52 \%$ engineering students choose "helpful". It reveals that, for adoption of rhetorical structure into their own writings, engineering students comparatively perform the best, followed by science and then art students, but for application of language features, all the students are at similar level, art and science students showing a tendency to perform better than engineering students.

\section{B. Students' Capabilities Of Dealing with These Tasks (Questions15-21)}

First, when asked about the level of difficulties in finding out genre features through these tasks (Q 15-18), students choose 1 from 5 scales of "difficulties" (very difficult, difficult, medium level, easy, very easy). For the "very difficult" level, the response distributions for the 4 parts (arranged in the sequence of Introduction, Body, Conclusion and Abstract) are respectively $0,0,1 \%$ and 0 . For "difficult" level, they're 6\%,31\%, 4\% and 5\%. For "medium level", they're $42 \%, 45 \%, 48 \%$ and $31 \%$. For "easy" level, they're $47 \%, 21 \%, 38 \%$ and $42 \%$. For "very easy" level, they're $5 \%$, $3 \%, 9 \%$ and $22 \%$. Here the author notices 3 numbers: $31 \%$, $21 \%$ and $22 \%$. The percentage " $31 \%$ " for Body part remains the highest at the "difficult" level and " $21 \%$ " for Body part remains the lowest at the "easy" level, signifying that genre analysis tasks of Body part are comparatively more difficult for students, which probably results from the same reason mentioned under the 1 st research question. Abstract is the highest at the "very easy" level, probably because this is the shortest and most frequently written part for all students that they've already acquired some genre knowledge. So teachers could give more attention to rhetorical instruction of Body part and less on Abstract. Furthermore, data from the "medium" and "easy" level shows that most students are capable of performing these tasks.

Second, when asked the level of difficulties in finding out language signals for each move (Q19), the percentage distributions for these 4 sections are respectively $1 \%$ (very difficult), $15 \%$ (difficult), $47 \%$ (medium) , 34\% (easy) and $3 \%$ (very easy). It's shown that for most students these tasks are of medium level or not very difficult. In addition, when asked if they'll try to imitate and apply the genre knowledge they've noticed from sample reading into their own writings (Q 20), 54\% of them say they will and $32 \%$ sometimes will. Also, 54\% of them will imitate and adopt the language features they've noticed previously and $35 \%$ will sometimes do it (Q 21). That is, over half of them are able to apply what they've learned from sample reading analysis.

As to subject difference, firstly, about the students' level of difficulties in dealing with genre features through these tasks (Q15-18), 58\% of art students choose "easy" for Introduction, 55\% science students choose "medium" for Conclusion and the rest of the calculations concerning "medium", "easy" and "very easy" level from all students are below $50 \%$, but the percentage of both "very difficult" and "difficult" are below $50 \%$ as well. That is, students from art, science and engineering are not showing significant difference and all students could do it but a certain degree of difficulty exists, especially for Body part. Based on the author's observation and interview with some students, 2 reasons are considered. One is that the first year graduate students as novice academic writers remain new to genre knowledge, so sample analysis with specific coding marks required by the teacher seems not that easy. The other reason is language barrier. Engineering students say that although they have some discipline-specific genre knowledge, long sentence structure plus complex technical terms lead to ambiguity for move analysis. Secondly, about finding out language signals (Q19), 50\% of the engineering students choose "medium" level of difficulty, but the other figures are all below 50\%. In addition, for adoption of genre knowledge and language features (Q20, 21), over $50 \%$ of art and engineering students say they will do both. Over $50 \%$ of science students will adopt language features, while no more than half of them say they'll adopt rhetorical structures. To summarize, it's not as easy as the author has imagined having the students analyze structural moves and highlight language signals, but once they find out and notice both features, they're willing to adopt in their own construction. So teachers need to spend time on related instruction of sample analysis before carrying out tasks.

\section{Students' Opinions Towards These Tasks Adopted in English Paper Writing Course (Questions 22-25)}

First, when asked if move analysis is helpful for their mastery of rhetorical structure (Q 22), 36\% of them think it's "very helpful", 55\% think "helpful", only $8 \%$ think it's just so-so and there are no responses for "little help" or "no help at all". That is, $91 \%$ of them think it's helpful. Second, when asked if they agree with the learning strategy of "reading and writing" (Q 23), 48\% of them say they totally agree with it, $47 \%$ say they agree with it and only 5\% say it's just so-so. There're none who show disagreement. It's shown that a combination of reading and writing in these genre-analysis tasks are well accepted by the majority of students. Third, when asked if they like the students-oriented way of analyzing moves so as to obtain the rhetorical structure (Q $24), 42 \%$ of them totally agree with it, 53\% agree and only $5 \%$ think it's just ok. This result is similar to that of question 23, that is, students-oriented move analysis tasks are acceptable. Fourth, when asked if they'll take initiative in doing all these tasks by English teacher's requirements (Q $25), 52 \%$ of them say they totally do, $43 \%$ say they do, $5 \%$ say they do but sometimes turn to their own ways. It's seen that these students are willingly cooperative in doing these tasks.

As to subject difference, for Q22, all students think move analysis is helpful for their mastery of rhetorical structure (art 63\%, science 55\% and engineering 52\%). For Q23, more than half of art and engineering students "totally agree" with the strategy of "reading and writing", and over half of science students say they "agree". For Q24, 58\% of art and science students "agree" respectively with the studentsoriented way of analyzing moves to acquire rhetorical knowledge, and $48 \%$ of engineering students choose "totally 
agree" and "agree" respectively. For Q 25, 53\% of art and $52 \%$ of science students say they do take initiative in doing these tasks by the teacher's requirements and $57 \%$ of engineering students say they "totally do". To summarize, there're not remarkable variation in the students' attitude towards task performance and most of them take a serious attitude, cooperative with the teacher and willing to carry out these tasks, while engineering students showing comparatively higher motivation in learning.

\section{CONCLUSION AND PEDAGOGICAL IMPLICATIONS}

In conclusion, genre analysis tasks in the present study are helpful in raising Chinese EFL graduate learners' rhetorical consciousness as well as in construction of their own texts. For pedagogical practice, teachers need to give more rhetorical instruction on Body part due to its complexity nature and subject difference. Besides, syntactic difficulty prevents many students from finding out and adopting language features in their own construction, so attention needs to be given as well. Those engineering students who have an English publication requirement are more rhetorically conscious, so graduate students are more motivated by field experts than language teachers. Thus the cooperation between English teacher and field expert is needed to promote students' motivation. Statistics also show that most students are capable of performing these tasks, but a certain degree of difficulty exists for all students, especially for Body part. So in task-design, teachers need to take subject difference into consideration. Once the students find out the rhetorical and linguistic features, they're likely to adopt in their own construction. Engineering students perform better than art and science students in finding out rhetorical and linguistic features, as well in adoption of rhetorical features in their own construction but worse in linguistic adoption. So motivation and English proficiency affect the students' capability of task performance. Finally, most students take a serious and cooperative attitude towards these tasks with engineering students showing a stronger learning motivation, for most of whom, there's publication requirement.

Limitations exist in the present study. The participants' subject areas are not evenly distributed, that is, there're much more participants from engineering (56) than those from science (24) and art (19). Besides, within engineering, there are much more participants from Information Science and Technology (43) than those from Environmental Engineering (1) and Chemical Engineering (1). It's the same case with art and science. For future research, a more scientific and observable way is to have an evenly distributed number of participants from different subject areas. In addition, the data source of students' responses towards genre analysis tasks is singularly from the quantitative analysis of questionnaire with the teacher's observation log only as a complementary proof. Further research needs to be done on qualitative analysis of teachers' observation log, students' process log or students' texts so as to find out more details concerning students' responses and performance in doing genre analysis tasks.

\section{REFERENCES}

[1] Hyland, K. Genre pedagogy: Language, literacy and L2 writing instruction [J]. Journal of Second Language Writing, 2007, 16(3), 148-164.

[2] Kuteeva, M. Graduate Learners' Approaches to Genre-analysis Tasks: Variations across and within Four Disciplines [J]. English for Specific Purposes 2013, (32): 84-96.

[3] Swales, J. M. 1990. Genre Analysis: English in Academic and Research Settings [M]. Cambridge: Cambridge University Press.

[4] Cheng, A. 2007. Transferring generic features and re-contextualizing genre awareness: Understanding writing performance in the ESP genre-based literacy framework [J]. English for Specific Purposes, 2007, (26): 287-307.

[5] Wang, Wei. 2017. Learner characteristics in an EAP thesis-writing class: Looking into students' responses to genre-based instruction and pedagogical task [J]. English for Specific Purpose, 2017, (47): 52-60.

[6] Yang, Ruiying. Genre Analysis of Research Articles in English: Theory and Application [M]. Beijing: Science Publication, 2016: 238239.

[7] Wen, Qiufang. Applied Linguistics: Research Methods and Thesis Writing[M]. Beijing: Foreign Language Teaching and Research Press, 2001: 116-119. 